D.O.I.: $10.3895 / \mathrm{S} 1808-04482006000100008$

\title{
ANÁLISE DE FALHAS ATRAVÉS DA APLICAÇÃO DO FMEA E DA TEORIA GREY
}

\section{FAILURE ANALYSIS THROUGH FMEA APPLICATION AND GREY THEORY}

\author{
Fabiano Leal ; Alexandre Ferreira de Pinho ${ }^{2}$; Dagoberto Alves de Almeida ${ }^{3}$ \\ Universidade Federal de Itajubá - UNIFEI - Itajubá - Brasil \\ fleal@unifei.edu.br \\ Universidade Federal de Itajubá - UNIFEI - Itajubá - Brasil \\ pinho@unifei.edu.br \\ Universidade Federal de Itajubá - UNIFEI - Itajubá - Brasil \\ dagoberto@unifei.edu.br
}

\begin{abstract}
Resumo
Este artigo tem como objetivo analisar as causas de falhas potenciais através da avaliação do RPN (risk priority number), apresentado na literatura na abordagem do FMEA (Failure Mode and Effect Analysis). O RPN será determinado através da teoria Grey, contribuindo desta maneira para contornar alguns problemas apresentados na literatura a respeito do método tradicional de cálculo do RPN. O artigo apresentará uma descrição passo a passo desta técnica, aplicada ao processo de distribuição de energia elétrica. Para isto, foram analisadas algumas falhas identificadas em uma distribuidora. Por fim, a análise considerou uma ponderação diferente para os fatores do FMEA, evidenciando as possibilidades de análise desta técnica.
\end{abstract}

Palavras-chave: Análise de Falhas, FMEA, Teoria Grey, Gestão da Manutenção.

\section{Introdução}

De acordo com Rausand e Oien (1996), a falha representa um conceito fundamental para a análise de confiabilidade, sendo a falha definida como o término da habilidade de um item para o desempenho de uma requerida função. A qualidade de uma análise de confiabilidade depende fortemente da habilidade do analista em identificar todas as funções desempenhadas pelos componentes e as possíveis falhas com potencial de ocorrência.

O FMEA (Failure Mode and Effect Analysis) pode ser considerado como uma importante técnica para a análise de falhas (STAMATIS, 2003). Através de três fatores (ocorrência, detecção e severidade), é realizada uma hierarquização de acordo com o risco potencial, representado no 
FMEA através do RPN (Risk Priority Number). Porém, alguns autores (Gilchrist, 1993; Ben-Daya e Raouf, 1996; Deng, 1989; Chang et al., 2001) criticam a forma de construção do RPN. Este artigo sugere uma maneira alternativa de construção do RPN, através da aplicação da Teoria Grey, desenvolvida por Chang et al. (2001), evidenciando uma opção para a hierarquização de ações de manutenção de acordo com os riscos potenciais, através de informações coletadas em uma distribuidora de energia elétrica do Brasil.

\section{Gestão de falhas no setor elétrico}

A distribuição de energia elétrica pode ser considerada um dos serviços de maior importância para a população, sendo a energia um dos recursos básicos ao desenvolvimento. De acordo com Monticelli e Garcia (2003), a grande motivação das empresas distribuidoras de energia deverá ser a redução dos custos para o consumidor final e a melhora da rentabilidade sem perda da qualidade e da confiabilidade do serviço prestado.

De acordo com Ganim (2003), através de alguns indicadores (Duração Equivalente de Interrupção por Unidade Consumidora - DEC; Freqüência Equivalente de Interrupção por Unidade Consumidora - FEC; Duração de Interrupção Individual por Unidade Consumidora - DIC; Duração Máxima de Interrupção Contínua por Unidade Consumidora - DMIC; Freqüência de Interrupção Individual por Unidade Consumidora - FIC), a ANEEL (Agência Nacional de Energia Elétrica) exerce um controle rigoroso sobre as concessionárias. Este controle tem por objetivo garantir a qualidade da energia, ou seja, incentivar as distribuidoras de energia a evitarem ao máximo a ocorrência de falhas.

Segundo o Comitê de Distribuição (1982), falha em sistemas de distribuição é todo evento que produz a perda de capacidade de um componente ou sistema desempenhar sua função, levandoos à condição de operação inadmissível. Dentre as falhas no fornecimento de energia elétrica, a interrupção do fornecimento de energia apresenta alta gravidade e influencia negativamente os índices de continuidade.

A falha pode atingir grandes proporções se afetar indústrias que tenham sua produção comprometida e que possam mover processos judiciais, implicando em penalizações financeiras contra a concessionária. Piores ainda são os casos em que as interrupções no fornecimento de energia possam afetar a vida humana, por exemplo, se a energia for interrompida para um hospital ou para a residência de um indivíduo que utilize um aparelho de sobre-vida, como um pulmão artificial (ALMEIDA et al., 2004). 


\section{O FMEA}

O FMEA (Failure Mode and Effect Analysis) é uma técnica de engenharia utilizada para definir, identificar e eliminar falhas conhecidas ou potenciais, de sistemas, projetos, processos e/ou serviços, antes que estas atinjam o cliente (STAMATIS, 2003).

Segundo Puente et al. (2002), o FMEA inicialmente foi utilizado pela Nasa (National Aeronautics and Space Administration) em 1963, e então expandido para a indústria automobilística, onde foi utilizado para quantificar e ordenar possíveis defeitos potenciais no estágio de projeto de produtos, antes de chegarem ao consumidor final, através de sessões de brainstormings que buscam levantar falhas que podem ocorrer.

Ainda segundo Puente et al. (2002), o FMEA basicamente consiste em dois estágios. Durante o primeiro estágio, possíveis modos de falhas de um produto ou processo e seus efeitos prejudiciais são identificados. Durante o segundo estágio, os times de engenheiros que trabalharam com o FMEA determinam o nível crítico (pontuação de risco) destas falhas e as colocam em ordem. A falha mais crítica será a primeira do ranking, e será considerada prioritária para a aplicação de ações de melhoria.

Há três fatores utilizados no FMEA que auxiliam na definição de prioridades de falhas. São eles: ocorrência $(\mathrm{O})$, severidade (S) e detecção (D). A ocorrência define a freqüência da falha, enquanto a severidade corresponde à gravidade do efeito da falha. A detecção é a habilidade para detectar a falha antes que ela atinja o cliente.

Para se avaliar a criticidade das causas de uma possível falha, é utilizado o RPN, sendo composto do produto dos três fatores do FMEA: D, O e S. Tendo obtido o RPN, as causas das falhas são ranqueadas, direcionando a atuação do gestor. No entanto, a literatura apresenta algumas críticas quanto à forma de obtenção do RPN (Gilchrist, 1993; Ben-Daya e Raouf, 1996; Deng, 1989; Chang et al., 2001). Estas críticas podem ser citadas a seguir:

- Não há uma regra algébrica precisa que determine uma pontuação para o índice de ocorrência detecção, como uma pontuação tradicional baseada em probabilidade de ocorrência e não detecção. A probabilidade é convertida em outro sistema de pontuação. Outro problema apontado é o cálculo do produto de três fatores, onde o fator $\mathrm{D}$, por exemplo, apresenta uma pontuação que segue uma escala linear, o que não ocorre com o fator ocorrência.

- Diferentes pontuações para O e D, por exemplo, poderiam resultar no mesmo resultado RPN, ainda que o risco envolvido seja completamente diferente.

a) - O RPN clássico não supri a possibilidade de considerar pesos de importância nos fatores $D, S$ e O. 
As tabelas a seguir (Tabelas 1, 2 e 3) apresentam um sistema de pontuação a ser utilizado na determinação dos fatores ocorrência, severidade e detecção, utilizadas em trabalhos da área (STAMATIS, 2003; CHANG et al., 2001). Para tanto, este artigo fará uso destes valores.

Tabela 1 - Critério de análise e sistema de ranqueamento para a ocorrência da falha

\begin{tabular}{|c|c|c|}
\hline Probabilidade de falha & Possíveis taxas de falhas & Rank \\
\hline Extremamente alta: quase inevitáveis & $\geq 1$ em 2 & 10 \\
\hline Muito alta & $1 \mathrm{em} 3$ & 9 \\
\hline Repetidas falhas & $1 \mathrm{em} 8$ & 8 \\
\hline Alta & 1 em 20 & 7 \\
\hline Moderadamente alta & $1 \mathrm{em} 80$ & 6 \\
\hline Moderada & $1 \mathrm{em} 400$ & 5 \\
\hline Relativamente baixa & 1 em 2000 & 4 \\
\hline Baixa & $1 \mathrm{em} 15000$ & 3 \\
\hline Remota & $1 \mathrm{em} 150000$ & 2 \\
\hline Quase impossível & 1 em 1500000 & 1 \\
\hline
\end{tabular}

Fonte: adaptado de Ford Motor Company (1988)

Tabela 2 - Critério de análise e sistema de ranqueamento para a detecção da causa da falha

\begin{tabular}{|l|l|c|}
\hline \multicolumn{1}{|c|}{ Deteç̧ão } & $\begin{array}{l}\text { Probabilidade de deteção da causa } \\
\text { da falha }\end{array}$ & 10 \\
\hline Absolutamente incerta & $\begin{array}{l}\text { A manutenção não detecta a causa da } \\
\text { falha potencial, ou não existe } \\
\text { manutenção }\end{array}$ & 9 \\
\hline Muito remota & $\begin{array}{l}\text { Chance muito remota de se detectar a } \\
\text { causa da falha }\end{array}$ & 8 \\
\hline Remota & $\begin{array}{l}\text { Chance remota de se detectar a causa } \\
\text { da falha }\end{array}$ & 7 \\
\hline Muito baixa & $\begin{array}{l}\text { Chance muito baixa de se detectar a } \\
\text { causa da falha }\end{array}$ & 6 \\
\hline Baixa & $\begin{array}{l}\text { Chance baixa de se detectar a causa } \\
\text { da falha }\end{array}$ & 5 \\
\hline Moderada & $\begin{array}{l}\text { Moderada chance de se detectar a } \\
\text { causa da falha }\end{array}$ & 4 \\
\hline Moderadamente alta & $\begin{array}{l}\text { Moderadamente alta a chance de se } \\
\text { detectar a causa da falha }\end{array}$ & 3 \\
\hline Alta & $\begin{array}{l}\text { Chance alta de se detectar a causa da } \\
\text { falha }\end{array}$ & $\begin{array}{l}\text { Chance muito alta de se detectar a } \\
\text { causa da falha }\end{array}$ \\
\hline Muito alta & $\begin{array}{l}\text { A manutenção quase certamente } \\
\text { detectará a causa da falha }\end{array}$ & 2 \\
\hline Quase certa & & 1 \\
\hline
\end{tabular}

Fonte: adaptado de Ford Motor Company (1988) 
Tabela 03 - Critério de análise e sistema de ranqueamento para a severidade (gravidade) dos efeitos da falha

\begin{tabular}{|c|c|c|}
\hline Efeito & Severidade do efeito & Rank \\
\hline Perigoso & $\begin{array}{l}\text { Falha é perigosa, e ocorre sem aviso. } \\
\text { Capaz de suspender a operação dos } \\
\text { sistemas e/ou envolve aspectos não } \\
\text { complacentes com regulações } \\
\text { governamentais }\end{array}$ & 10 \\
\hline Sério & $\begin{array}{l}\text { Os efeitos podem ser perigosos e/ou } \\
\text { envolvem aspectos não complacentes } \\
\text { com regulações governamentais }\end{array}$ & 9 \\
\hline Importante & $\begin{array}{l}\text { Produto inoperável, com perda da } \\
\text { função básica. Sistema inoperante }\end{array}$ & 8 \\
\hline Impactante & $\begin{array}{l}\text { Desempenho do produto sofre } \\
\text { impacto. Sistema pode não operar }\end{array}$ & 7 \\
\hline Significativo & $\begin{array}{l}\text { Desempenho do produto é degradado. } \\
\text { Funções atreladas ao conforto podem } \\
\text { não operar. }\end{array}$ & 6 \\
\hline Moderado & $\begin{array}{l}\text { Moderado efeito no desempenho do } \\
\text { produto. Produto requer reparos. }\end{array}$ & 5 \\
\hline Baixo & $\begin{array}{l}\text { Pequeno efeito no desempenho do } \\
\text { produto. O produto não requer } \\
\text { reparos. }\end{array}$ & 4 \\
\hline Desprezível & $\begin{array}{l}\text { Efeito desprezível no desempenho do } \\
\text { produto ou sistema. }\end{array}$ & 3 \\
\hline Muito desprezível & $\begin{array}{l}\text { Efeito muito desprezível no } \\
\text { desempenho do produto ou sistema }\end{array}$ & 2 \\
\hline Nenhum & Nenhum efeito & 1 \\
\hline
\end{tabular}

Fonte: adaptado de Ford Motor Company (1988)

\section{Uso da teoria Grey na determinação do RPN}

Algumas pesquisas tem sido realizadas com o objetivo de desenvolver uma nova relação entre os fatores do FMEA, através do cálculo do RPN (Gilchrist 1993; Ben-Daya e Raouf, 1993; Chang et al., 2001). Entre estas propostas, apresenta-se a teoria grey, proposta por Deng (1989). Esta teoria permite uma mensuração para analisar a relação entre séries qualitativas e quantitativas discretas, e todos os componentes das séries devem estar conforme algumas características prédefinidas (Chang et al., 2001).

Segundo Chen e Ting (2002), a relação grey corresponde a uma relação incerta entre coisas ou fatores de um sistema, ou quando o efeito do fator sobre o comportamento principal é obscuro. A análise da relação grey analisa e determina o grau de influência entre fatores ou simplesmente mensura a contribuição dos fatores no comportamento principal analisado. Chen e Ting (2002) utilizaram a teoria grey para avaliar a importância de vários fatores na qualidade de serviços prestados, como tangibilidade, confiabilidade, empatia, acessibilidade, entre outros. 
Chang et al. (2001) propõem a aplicação da teoria grey através de alguns passos. Este artigo irá representar estes passos através de uma aplicação direta nos fatores do FMEA, como mostra a Figura 1.

Figura 1 - Cada causa potencial i é caracterizada pelos fatores Oi (ocorrência da causa i), Di (detecção da causa i) e Si (severidade da causa i)

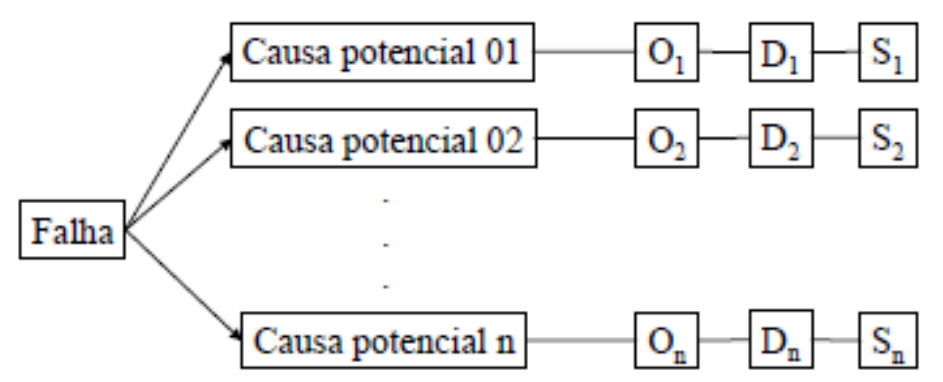

A aplicação da teoria grey para o cálculo do FMEA pode-se dar da seguinte forma:

a) Passo 01- Estabelecer uma série comparativa: esta série é apresentada na forma de matriz, onde $n$ representa o total de causas associadas à falha.

b)

$$
\mathrm{X}=\left[\begin{array}{ccc}
\mathrm{O}_{1} & \mathrm{D}_{1} & \mathrm{~S}_{1} \\
\mathrm{O}_{2} & \mathrm{D}_{2} & \mathrm{~S}_{2} \\
\cdot & \cdot & \cdot \\
\cdot & \cdot & \cdot \\
\mathrm{O}_{\mathrm{n}} & \mathrm{D}_{\mathrm{n}} & \mathrm{S}_{\mathrm{n}}
\end{array}\right]
$$

c) Passo 02 - Estabelecer uma série padrão: uma série padrão é desenvolvida através dos menores valores dos fatores do FMEA registrados nas tabelas de referência (Tabelas 1, 2 e 3).

d) Passo 03 - Obter a diferença entre a série comparativa e a série padrão: a diferença entre os valores dos fatores do FMEA e da série padrão deve ser determinada e expressa em uma matriz.

$$
\mathrm{D}=\left[\begin{array}{ccc}
\Delta \mathrm{O}_{1} & \Delta \mathrm{D}_{1} & \Delta \mathrm{S}_{1} \\
\Delta \mathrm{O}_{2} & \Delta \mathrm{D}_{2} & \Delta \mathrm{S}_{2} \\
\cdot & \cdot & \cdot \\
\cdot & \cdot & \cdot \\
\Delta \mathrm{O}_{\mathrm{n}} & \Delta \mathrm{D}_{\mathrm{n}} & \Delta \mathrm{S}_{\mathrm{n}}
\end{array}\right]
$$

e) Passo 04 - Obter os coeficientes de relação: os fatores do FMEA devem ser comparados com a série padrão. A máxima e a mínima diferença obtida no terceiro passo devem ser registradas, 
para a obtenção do coeficiente de relação. Dois passos devem ser utilizados: definição da constante $\beta$ e definição dos coeficientes $\gamma$, sendo i o número da causa potencial, podendo variar de 1 até $n$.

$$
\begin{gathered}
\beta=\Delta_{\mathrm{MDN}}+\xi \Delta_{\mathrm{MAX}} \\
\gamma \mathrm{O}_{\mathrm{i}}=\frac{\beta}{\Delta \mathrm{O}_{\mathrm{i}}+\xi \Delta_{\mathrm{MAX}}} \\
\gamma \mathrm{D}_{\mathrm{i}}=\frac{\beta}{\Delta \mathrm{D}_{\mathrm{i}}+\xi \Delta_{\mathrm{MAX}}} \\
\gamma \mathrm{S}_{\mathrm{i}}=\frac{\beta}{\Delta \mathrm{S}_{\mathrm{i}}+\xi \Delta_{\mathrm{MAX}}}
\end{gathered}
$$

Segundo Chang et al. (2001), o identificador $\xi$ pode assumir um valor entre 0 e 1 , geralmente assumindo o valor 0,5. Este identificador apenas afeta o valor relativo do risco, sem alterar a prioridade, como será visto adiante.

f) Passo 05 - Determinação do grau de relação: antes da determinação do grau de relação, os pesos relativos aos fatores ocorrência (O), detecção (D) e severidade (S) devem ser decididos. O grau de relação é definido como:

onde:

$$
\begin{gathered}
\mathrm{C}_{\mathrm{i}}=\gamma \mathrm{O}_{\mathrm{i}} \cdot \alpha_{\mathrm{O}}+\gamma \mathrm{D}_{\mathrm{i}} \cdot \alpha_{\mathrm{D}}+\gamma \mathrm{S}_{\mathrm{i}} \cdot \alpha_{\mathrm{S}} \\
\alpha_{0}+\alpha_{\mathrm{D}}+\alpha_{\mathrm{S}}=1
\end{gathered}
$$

Segundo Chang et al. (2001), o FMEA tradicional não atribui pesos diferentes aos fatores ocorrência, detecção e severidade. Porém, esta possibilidade permite uma análise diferenciada e customizada ao processo.

g) Passo 06 - Ranquear as prioridades de risco: a hierarquia será construída a partir do grau de relação. O grau de relação denota a relação entre a pontuação da causa potencial e os valores ótimos dos fatores de decisão. Quanto maior o grau de relação, menor o efeito da causa. Ou seja, serão priorizadas as causas com menores graus de relação. 


\section{Caso analisado}

Para aplicação da técnica, foram analisadas algumas situações que provocam a interrupção do fornecimento de energia a consumidores. Estas informações foram levantadas em uma distribuidora brasileira de energia elétrica, através de brainstormings, onde funcionários de diversos departamentos apontaram causas e as classificaram de acordo com os fatores do FMEA (ocorrência, detecção e severidade). De acordo com as Tabelas 1, 2 e 3 deste trabalho chegou-se aos dados preliminares apresentados na Tabela 4. As causas potenciais foram registradas de acordo com a nomenclatura utilizada pelos funcionários da distribuidora.

Tabela 4 - Determinação dos fatores O, D e S para cada causa potencial i identificada

\begin{tabular}{|l|c|c|c|}
\hline \multicolumn{1}{|c|}{ Causa potencial (i) } & Ocorrência (O) & Detecção (D) & Severidade (S) \\
\hline 1- Cabo partido & 6 & 7 & 3 \\
\hline 2- Má atuação do religador & 4 & 9 & 8 \\
\hline 3- Queima de elo fusível & 7 & 1 & 1 \\
\hline 4- Queima de trafo & 6 & 7 & 1 \\
\hline 5- Problema de mufla & 5 & 1 & 5 \\
\hline
\end{tabular}

A partir destes dados, irá se determinar a priorização de ações, segundo a aplicação da teoria grey.

a) Passo 01 - Estabelecimento de uma série comparativa, apresentada na forma de matriz.

$$
\mathrm{X}=\left[\begin{array}{lll}
\mathrm{O} & \mathrm{D} & \mathrm{S} \\
6 & 7 & 5 \\
4 & 1 & 3 \\
7 & 9 & 8 \\
6 & 1 & 1 \\
5 & 7 & 1
\end{array}\right]
$$

b) Passo 02 - Estabelecimento de uma série padrão

$$
\text { Série }=\left[\begin{array}{lll}
1 & 1 & 1
\end{array}\right]
$$

c) Passo 03 - Obtenção da diferença entre a série comparativa e a série padrão. 
d) Passo 04 - Obter os coeficientes de relação, através da definição da constante $\beta$ e coeficientes $\gamma$.

$$
\begin{array}{ccc}
\beta=0+0,5 \cdot 8 \Rightarrow \beta=4 \\
\gamma \mathrm{O}_{1}=0,444 & \gamma \mathrm{D}_{1}=0,400 & \gamma \mathrm{S}_{1}=0,500 \\
\gamma \mathrm{O}_{2}=0,571 & \gamma \mathrm{D}_{2}=1,000 & \gamma \mathrm{S}_{2}=0,667 \\
\gamma \mathrm{O}_{3}=0,400 & \gamma \mathrm{D}_{3}=0,333 & \gamma \mathrm{S}_{3}=0,364 \\
\gamma \mathrm{O}_{4}=0,444 & \gamma \mathrm{D}_{4}=1,000 & \gamma \mathrm{S}_{4}=1,000 \\
\gamma \mathrm{O}_{5}=0,500 & \gamma \mathrm{D}_{3}=0,400 & \gamma \mathrm{S}_{3}=1,000
\end{array}
$$

e) Passo 05 - Determinação do grau de relação, considerando os pesos relativos aos fatores ocorrência (O), detecção (D) e severidade (S). Neste passo, ir-se-á considerar inicialmente uma análise onde não serão atribuídos pesos diferentes aos fatores. A Tabela 5 representa todos os $\mathrm{C}_{\mathrm{i}}$, para as cinco causas potenciais analisadas. Neste caso, tem-se o seguinte:

$$
\begin{aligned}
& C_{1}=\frac{0,444+0,400+0,500}{3} \\
& C_{1}=0,448
\end{aligned}
$$

f) Passo 06 - Ranquear as prioridades de risco, a partir do grau de relação. Quanto maior o grau de relação, menor o efeito da causa. Ou seja, serão priorizadas as causas com menores graus de relação, conforme mostra a Tabela 5 .

Tabela 5 - Grau de relação $C_{i}$ de cada causa i, e sua respectiva priorização

\begin{tabular}{|l|c|c|}
\hline \multicolumn{1}{|c|}{ Causa potencial (i) } & $\mathbf{C}_{\mathbf{i}}$ & Priorização \\
\hline 1- Cabo partido & 0,463 & 2 \\
\hline 2- Má atuação do religador & 0,705 & 3 \\
\hline 3- Queima de elo fusível & 0,368 & 1 \\
\hline 4- Queima de trafo & 0,833 & 5 \\
\hline 5- Problema de mufla & 0,730 & 4 \\
\hline
\end{tabular}

Para a análise da priorização destas cinco causas, foi elaborado um possível cenário onde o gestor decide por ponderar os fatores. Será considerada uma ponderação maior ao fator severidade, 
objetivando dedicar uma atenção especial a causas com severidades maiores. Foram estabelecidos os seguintes pesos: $\alpha_{\mathrm{O}}$ (peso do fator ocorrência) igual a 0,$3 ; \alpha_{\mathrm{D}}$ (peso do fator detecção) igual a 0,2 e $\alpha_{\mathrm{S}}$ (peso do fator severidade) igual a 0,5. Novos graus de relação, representados na Tabela 6, foram encontrados, alterando assim a priorização de ações.

Tabela 6 - Novos graus de relação $\mathrm{C}_{\mathrm{i}}$ e sua respectiva priorização, após a ponderação dos fatores $\mathrm{O}$, D e S.

\begin{tabular}{|l|c|c|}
\hline \multicolumn{1}{|c|}{ Causa potencial (i) } & $\mathbf{C}_{\mathbf{i}}$ & Priorização \\
\hline 1- Cabo partido & 0,463 & 2 \\
\hline 2- Má atuação do religador & 0,705 & 3 \\
\hline 3- Queima de elo fusível & 0,368 & 1 \\
\hline 4- Queima de trafo & 0,833 & 5 \\
\hline 5- Problema de mufla & 0,730 & 4 \\
\hline
\end{tabular}

Neste caso, o gestor pode influenciar a hierarquização de ações de acordo com o peso associado aos fatores do FMEA. Vale ressaltar que a grande contribuição deste tipo de análise é criar uma rotina de avaliação onde, a partir da entrada de novos dados, novos cenários possam ser gerados, evidenciando problemas que de alguma forma afetam o processo normal de uma empresa.

\title{
6. Conclusões
}

Através de informações coletadas em uma distribuidora de energia elétrica, este artigo apresentou os resultados obtidos através da hierarquização dos riscos potenciais através do FMEA a da Teoria Grey. Cada etapa da análise foi evidenciada através de passos descritos no presente trabalho.

Os resultados obtidos foram comparados com uma nova análise, onde se ponderou de maneira mais relevante o fator severidade, influenciando desta maneira o risco potencial (RPN). Neste caso, a causa "Má atuação do religador" alcançou uma posição acima na hierarquia de riscos potenciais.

Vários cenários podem ser criados, de acordo com as ponderações utilizadas, objetivando direcionar recursos priorizando problemas com maiores potenciais de risco ao sistema em análise.

\begin{abstract}
This article aims to analyze the potential failure causes through the evaluation of RPN (risk priority number), presented FMEA (Failure Mode and Effect Analysis) approach. The RPN will be determined through the Grey theory. This article will present a detailed technique description, applied to the energy electric distribution. Some failures identified in a company were analyzed. Finally, this approach considered a different analysis for the FMEA factors.
\end{abstract}

Key word: failure analysis, FMEA, Grey Theory, Maintenance Management. 


\section{Referências}

ALMEIDA, D.A.de; LEAL, F. e ALMEIDA, R.A.de. Mapeamento do processo de formação de falhas: uma aplicação no setor elétrico. In. CONGRESSO BRASILEIRO DE ENERGIA, 10., Rio de Janeiro, 2004.

BEN-DAYA, M. e RAOUF, A. A revised failure mode and effects analysis model. International Journal of Quality Reliability Management, n. 1, v. 13, 1993.

CHANG, C.L.; LIU, P.H. e WEI, C.C. Failure mode and effects analysis using grey theory. Integrated Manufacturing Systems, n.3, v.12, 2001.

cross ${ }^{\text {ref }}$

CHEN, C.N. \& TING, S.C. A study using the grey system theory to evaluate the importance of various service quality factors. International Journal of Quality \& Reliability Management, n. 7, v. 19, 2002.

cross ${ }^{\text {ref }}$

COMITE DE DISTIBUIÇAO. Planejamento de Sistemas de Distribuição. Rio de Janeiro: Editora Campus, $1{ }^{a}$ Edição, v. 1, 1982.

DENG, J. Introduction to grey system theory. Journal of Grey System, n.1, v.1, 1989.

FORD MOTOR COMPANY. Potential Failure Mode and Effects Analysis (FMEA). Reference Manual, 1988.

GANIM, A. Setor Elétrico Brasileiro: Aspectos regulamentares e tributários. Editora Canalenergia, 2003.

GILCHRIST, W. Modeling failure modes and effects analysis. International Journal of Quality Reliability Management, n. 5, v. 10, 1993.

cross'

MONTICELLI, A. \& GARCIA, A. Introdução a sistemas de energia elétrica. Campinas, SP: Editora da Unicamp, 2003.

PUENTE, J.; PINO, R.; PRIORE, P. \& LA FUENTE, D. de. A decision support system for applying failure mode and effects analysis. International Journal of Quality \& Reliability Management, n.2, v. 19, 2002.

cross ${ }^{\text {ref }}$

RAUSAND, M. \& OIEN, K. The basic concepts of failure analysis. Reliability Engineering and System Safety, 1996.

STAMATIS, D.H. Failure Mode and Effect Analysis: FMEA from theory to execution. Milwaukee, Winsconsin: ASQ Quality Press, second edition, 2003.

\section{Dados completos do primeiro autor:}

Nome completo: Fabiano Leal

Filiação institucional: UNIFEI - Itajubá MG.

Função ou cargo ocupado: Professor

Endereço completo para correspondência (bairro, cidade, estado, país e CEP):

Av. BPS, 182 Apto. 403, Centro - Itajubá - MG

CEP: $37500-176$

Telefones para contato: (35) 8828-3622

E-mail: fleal@unifei.edu.br 
Aceito para publicação em: 16/01/2006 